\title{
SRY-related HMG box-2 role in anaplastic thyroid cancer aggressiveness is related to the fibronectin 1 and PI3K/AKT pathway
}

\author{
PENG WANG, JINBIAO SHANG, JIANQIANG ZHAO, KEJING WANG, \\ LIANG GUO, JIALEI GU and WENDONG WANG
}

\begin{abstract}
Department of Head and Neck Surgery, Institute of Cancer Research and Basic Medical Sciences of Chinese Academy of Sciences, Cancer Hospital of University of Chinese Academy of Sciences, Zhejiang Cancer Hospital, Hangzhou, Zhejiang 310022, P.R. China
\end{abstract}

Received July 4, 2019; Accepted November 11, 2019

DOI: $10.3892 / \mathrm{mmr} .2020 .10907$

\begin{abstract}
Anaplastic thyroid cancer (ATC) is a rare thyroid tumor associated with high mortality rates; thus, the identification of novel molecular targets and the development of therapeutic strategies are urgently required. The present study aimed to investigate the role of SRY-related HMG box-2 (SOX2) in ATC cells and explore whether the underlying mechanism was associated with fibronectin 1 (FN1). The proliferative, migratory and invasive ability of ATC cell lines was investigated using Cell Counting Kit-8, colony formation, wound-healing and Transwell assays, respectively; SOX2 expression in FRO cells was knocked down using small interfering RNA and SOX2 overexpression in FRO cells was achieved using cDNA constructs; and reverse transcription-quantitative PCR and western blotting were used to identify the mechanism of action underlying the SOX-2 mediated increased in cell aggressive phenotypes. Increased protein expression levels of SOX 2 protein were observed in ATC tissue, and FRO and 8505c ATC cell lines. SOX2 overexpression increased the cell viability, and proliferative, migratory and invasive abilities of FRO cell lines. SOX2 overexpression increased FN1, p65, phosphorylated PI3K and AKT expression levels, whereas the knockdown of SOX2 promoted the opposite effects. In conclusion, the present study suggested a possible model of SOX2-mediated gene regulation in ATC cells, in which the overexpression of SOX2 promoted FN1 expression via the PI3K/AKT signaling pathway to
\end{abstract}

Correspondence to: Dr Wendong Wang, Department of Head and Neck Surgery, Institute of Cancer Research and Basic Medical Sciences of Chinese Academy of Sciences, Cancer Hospital of University of Chinese Academy of Sciences, Zhejiang Cancer Hospital, 38 Guangji Road, Hangzhou, Zhejiang 310022, P.R. China E-mail: wendongwangopj@163.com

Key words: SRY-related HMG box-2, anaplastic thyroid cancer, fibronectin 1 induce the aggressive phenotype of ATC. These findings may provide crucial molecular insights into ATC pathogenesis and may demonstrate potential to develop into novel therapeutic interventions for patients with ATC.

\section{Introduction}

Thyroid cancer is a common endocrine malignancy, accounting for $2.1 \%$ of all cancers worldwide (1); it includes four major types: Papillary, medullary, follicular and anaplastic thyroid cancer (ATC) (2). Among them, ATC is a rare form of thyroid cancer, comprising $<2 \%$ of all thyroid cancers, but causing $>50 \%$ of thyroid-related mortality $(3,4)$. Most patients with ATC develop distant metastasis, which often makes traditional treatments ineffective (5); thus, there is an urgent requirement for effective diagnostic markers and therapeutic targets.

SRY-related HMG box-2 (SOX2) is a pluripotency-associated transcription factor, which is involved in growth, metastasis, tumorigenicity and drug resistance in multiple different types of cancer (6); for example, the expression of SOX2 may be implicated in the metastasis and invasion of pancreatic cancer (7) and gastric and prostatic carcinogenesis $(8,9)$. In addition, SOX2 has been observed to promote cell proliferation and tumorigenesis through inducing cell cycle arrest and apoptosis (10). SOX2 was previously found to be upregulated in ATC tissues, and the inhibition of SOX2 expression improved the sensitivity of ATC cell lines to antitumor drugs $(11,12)$. These investigations indicated that SOX2 may be involved in the growth of ATC cells; however, the mechanism remains largely unknown.

Fibronectin 1 (FN1) is an important regulatory factor of cell migration and metastasis, which is reported to be involved in the development of human cutaneous squamous cell carcinoma (13). FN1 was observed to be upregulated in thyroid cancer, and its silencing inhibited the proliferation, invasion and migration of thyroid cancer, indicating that FN1 may be involved in the development of thyroid cancer $(14,15)$. Furthermore, FN1 stimulated cell proliferation and inhibited apoptosis through the activation of PI3K and NF- $\mathrm{BB}(16)$. A previous study demonstrated that SOX2 targeted FN1 and mediated the migration and invasion of 
ovarian cancer cells (17); thus, it was hypothesized that SOX2 may also regulate the expression of FN1 to mediate cellular migration and invasion in ATC. The present study aimed to explore the role of SOX2 and FN1 and determine whether SOX2 affected the aggressive phenotype of ATC through affecting FN1 and PI3K/AKT signaling.

\section{Materials and methods}

Cell lines and culture. Human ATC cell lines FRO and 8505c and the normal human thyroid follicular epithelial cell line Nthy-ori 3-1 were obtained from the China Infrastructure of Cell Line Resources, Institute of Basic Medical Sciences, Chinese Academy of Medical Sciences. Cells were cultured in DMEM (Beyotime Institute of Biotechnology), supplemented with $10 \%$ FBS (Gibco; Thermo Fisher Scientific, Inc.), $100 \mathrm{U} / \mathrm{ml}$ penicillin and $100 \mu \mathrm{g} / \mathrm{ml}$ streptomycin, and maintained in a humidified atmosphere at $37^{\circ} \mathrm{C}$ with $5 \% \mathrm{CO}_{2}$.

Patient studies. The study was performed in accordance with the Declaration of Helsinki and approved by The Ethics Committee of Zhejiang Cancer Hospital (Hangzhou, China). Written informed consent was obtained from each participant prior to the study. Twenty pairs of surgical specimens (undifferentiated ATC tissue and para-carcinoma tissue) were collected from patients (11 females and 9 males; age range, 41-67 years; mean age, $51.25 \pm 10.20$ years) in Zhejiang Cancer Hospital (Hangzhou, China) between July 2018 and May 2019. Para-carcinoma tissue [negative control (NC)] was defined as tissue located $>0.5 \mathrm{~cm}$ away from the tumor. Patients $>18$ years of age with an ACT diagnosis were eligible for this study, whereas patients with other malignancies were excluded from this study. Tissue samples were collected, immediately frozen with liquid nitrogen and stored in a refrigerator at $-80^{\circ} \mathrm{C}$ until use.

Reverse transcription-quantitative PCR (RT- $q P C R)$. Total RNA was extracted from $1 \times 10^{6} \mathrm{FRO}, 8505 \mathrm{c}$ and Nthy-ori 3-1 cells, and $50 \mathrm{mg}$ ATC and para-carcinoma tissue samples using TRIzol ${ }^{\circledR}$ reagent (Invitrogen; Thermo Fisher Scientific, Inc.), according to the manufacturer's protocol. Total RNA was reverse transcribed into cDNA using the PrimeScript RT-PCR kit (Takara Biotechnology Co., Ltd.), according to the manufacturer's protocol. qPCR was subsequently performed in triplicate using the $\mathrm{SYBR}^{\circledR}{ }^{\circledR}$ reenER ${ }^{\mathrm{TM}}$ qPCR Supermix kit (Invitrogen; Thermo Fisher Scientific, Inc.), according to the manufacturer's protocol, and a StepOnePlus RT-PCR system (Thermo Fisher Scientific, Inc.). The following primer pairs were used for the qPCR: SOX2 forward, 5'-GTGGAAACT TTTGTCGGAGAC-3' and reverse, 5'-CGAGTAGGACAT GCTGTAGGT-3'; and GAPDH forward, 5'-ACAACTTTG GTATCGTGGAAGG-3' and reverse, 5'-GCCATCACGCCA CAGTTTC- 3 '. The following thermocycling conditions were used for the qPCR: Initial denaturation at $94^{\circ} \mathrm{C}$ for $15 \mathrm{~min}$; 38 cycles at $94^{\circ} \mathrm{C}$ for $30 \mathrm{sec}, 58^{\circ} \mathrm{C}$ for $30 \mathrm{sec}$ and $72^{\circ} \mathrm{C}$ for $30 \mathrm{sec}$; and a final extension at $72^{\circ} \mathrm{C}$ for $7 \mathrm{~min}$. SOX2 mRNA expression levels were quantified using the $2^{-\Delta \Delta C q}$ method (18) and GAPDH was used as the internal loading control.

Immunohistochemistry (IHC) analysis. IHC was performed using the standard streptavidin-peroxidase method (19). Tissue samples were fixed with $10 \%$ formalin fixative for $24 \mathrm{~h}$ at room temperature, embedded into paraffin and prepared into 4- $\mu \mathrm{m}$ sections. The sections were subsequently deparaffinized in xylene at $92^{\circ} \mathrm{C}$ and rehydrated in a descending alcohol series. Following antigen retrieval, endogenous peroxidase activity was blocked using $3 \% \mathrm{H}_{2} \mathrm{O}_{2}$ for $10 \mathrm{~min}$ at $22^{\circ} \mathrm{C}$. Sections were then blocked with 5\% goat serum (Thermo Fisher Scientific, Inc.) for $1 \mathrm{~h}$ at room temperature and subsequently incubated overnight at $4{ }^{\circ} \mathrm{C}$ with primary antibodies against SOX2 (1:300; cat. no. sc-365823; Santa Cruz Biotechnology, Inc.). Primary antibodies were substituted with $0.2 \mathrm{~mol} / \mathrm{l} \mathrm{PBS} \mathrm{(pH} \mathrm{7.4)} \mathrm{for}$ the negative control. Following the primary antibody incubation, the sections were incubated with a biotinylated horse anti-mouse IgG secondary antibody (1:100; cat. no. BA-2001; Vector Laboratories; Maravai Life Sciences) at room temperature for $30 \mathrm{~min}$, followed by incubation with a streptavidin horseradish peroxidase complex (1:5,000; cat. no. SA-5004; Vector Laboratories; Maravai Life Sciences) at room temperature for $20 \mathrm{~min}$. Finally, sections were counterstained with hematoxylin for $3 \mathrm{~min}$ at room temperature. Chromogen detection was performed using a Tris- $\mathrm{HCl}$ solution containing 0.02\% 3,3'-diaminobenzidine (Dako; Agilent Technologies, Inc.) for $5 \mathrm{~min}$ at room temperature. Stained tissue was visualized using a light microscope (magnification, x200; Olympus Corporation). Positive staining was defined as $>10 \%$ of cells appearing as brown granules and was quantified using ImageJ version 1.37 software (National Institutes of Health).

Cell transfection. Cell transfection and small interfering (si)RNA interference were performed using the Lipofectamine ${ }^{\circledR}$ 2000 Transfection kit (Invitrogen; Thermo Fisher Scientific, Inc.). A total of $4 \times 10^{5} \mathrm{FRO}$ cells were seeded into 6-well plates and cultured to $90-95 \%$ confluence. A pcDNA3.1 plasmid ( $4 \mu \mathrm{g}$; Invitrogen; Thermo Fisher Scientific, Inc.) carrying a SOX2 cDNA insert (pcDNA3.1-SOX2) was used to construct FRO cells overexpressing SOX2, and the empty pcDNA3.1 $(4 \mu \mathrm{g})$ plasmid was used as the NC. The amplification of SOX2 expression used the following primers: Forward, 5'-GGAGACCCAAGCTGG CTAGCATGTACAACATGATGGAGACGG-3' and reverse, 5'-GTTTAAACGGGCCCTCTAGATCACATGTGTGAGAGG GGC-3'. Genetic knockdown of SOX2 was performed using $2 \mu \mathrm{g}$ siRNA against SOX2 (siSOX2) and $2 \mu \mathrm{g}$ control non-targeting siRNA (siNC) was used as the NC. The siNC sequence was 5'-UUCUCCGAACGUGUCACGUTT-3'; and the siSOX2 sequence was 5'-CAGGTTGATATCGTTGGTAAT-3'. Cells were transfected for $48 \mathrm{~h}$ prior to subsequent experimentation and the overexpression or inhibition of the expression of SOX2 was confirmed using western blotting following transfection.

Western blotting. Total protein was extracted from $1 \times 10^{4}$ FRO cells/well using RIPA lysis buffer (Beyotime Institute of Biotechnology). Total protein was quantified using a bicinchoninic acid assay and $30 \mu \mathrm{g}$ protein/lane was separated by $10 \%$ SDS-PAGE. The separated proteins were subsequently transferred onto a PVDF membrane (EMD Millipore) and blocked for $2 \mathrm{~h}$ at $25^{\circ} \mathrm{C}$ in $5 \%$ non-fat milk. The membranes were incubated with the following primary antibodies at $4^{\circ} \mathrm{C}$ overnight: Anti-SOX2 (1:400; cat. no. sc-365823; Santa Cruz Biotechnology, Inc.), anti-FN1 (1:400; cat. no. sc-69681; Santa Cruz Biotechnology, Inc.), anti-p65 (1:400; cat. no. sc-8008; 
Santa Cruz Biotechnology, Inc.), anti-PI3K (1:500; cat. no. sc-1637; Santa Cruz Biotechnology, Inc.), anti-phosphorylated (p)-PI3K (1:1,000; cat. no. 4228S, Cell Signaling Technology, Inc.), anti-AKT (1:400; cat. no. sc-8312; Santa Cruz Biotechnology, Inc.), anti-p-AKT (1:400; cat. no. sc-135650; Santa Cruz Biotechnology, Inc.) and anti-GADPH (1:2,000; cat. no. sc-47724; Santa Cruz Biotechnology, Inc.). Following the primary incubation, membranes were incubated with horseradish peroxidase-conjugated anti-mouse or anti-rabbit secondary antibodies (1:5,000; cat. nos. sc-2357 and sc-2005; Santa Cruz Biotechnology, Inc.) for $2 \mathrm{~h}$ at $25^{\circ} \mathrm{C}$. Protein bands were visualized using enhanced chemiluminescence (Thermo Fisher Scientific, Inc.). Protein expression was quantified using Quantity One 1-D version 4.6.2 software (Bio-Rad Laboratories, Inc.), with GAPDH as the loading control.

Cell viability and proliferation assays. A total of $1 \times 10^{3}$ FRO cells/well were seeded into a 96-well plate and the cell viability was analyzed using a Cell Counting Kit-8 (CCK-8; Beyotime Institute of Biotechnology) assay, according to the manufacturer's protocol. Cell viability was measured at $48 \mathrm{~h}$ post-transfection at an absorbance of $450 \mathrm{~nm}$ using a $\mu$ Quant ${ }^{\mathrm{TM}}$ MQX200 microplate reader (Bio-Tek Instruments, Inc.).

A colony formation assay was performed based on the standard two-layer soft agar culture to assess proliferation. A total of 500 transfected FRO cells/well were cultured in DMEM in a humidified atmosphere at $37^{\circ} \mathrm{C}$ with $5 \% \mathrm{CO}_{2}$ for 12 days. Subsequently, cells were fixed with 1:3 acetic acid methanol at room temperature for $15 \mathrm{~min}$ and then stained with $0.1 \%$ crystal violet at room temperature for $30 \mathrm{~min}$. The colonies were visualized and manually counted using an Olympus CKX41 light microscope (magnification, x100; Olympus Corporation).

Wound healing assay. The migratory ability of transfected FRO cells was detected using a wound healing assay. A total of $2 \times 10^{5}$ FRO cells were seeded into a 6 -well plate and cultured in serum-free DMEM to $\geq 95 \%$ confluence. A $10-\mu$ l pipette tip was used to generate the wound in the monolayer cells and the cells were washed with serum-free medium. At the 0 and 24-h timepoint following wound induction, cells were visualized using an inverted light microscope (magnification, x100; Olympus Corporation). The relative migratory distance was calculated using the following formula: Relative migratory distance $(\%)=[($ width of cell wound at $0 \mathrm{~h}$-width of cell wound at $24 \mathrm{~h}$ )/width of cell wound at $0 \mathrm{~h}] \times 100$.

Matrigel invasion assay. Cell invasion was assessed using a Matrigel-coated Transwell chamber (BD Biosciences), according to the manufacturer's protocol (20). A total of $1 \times 10^{5}$ transfected FRO cells/well were plated in the upper chambers of Matrigel-coated $8-\mu \mathrm{m}$ Transwell plates in $100 \mu \mathrm{l}$ serum-free DMEM and incubated at $37^{\circ} \mathrm{C}$ and $5 \% \mathrm{CO}_{2}$. DMEM supplemented with $10 \%$ FBS as a chemoattractant was plated in the lower chambers. Following incubation for $24 \mathrm{~h}$ at $37^{\circ} \mathrm{C}$, the non-invasive cells were removed from the upper surface of the Matrigel using a cotton swab. The migratory cells on the underside of the membrane were fixed with cold methanol and acetic acid $(3: 1 \mathrm{v} / \mathrm{v})$ for $30 \mathrm{~min}$ at room temperature and subsequently stained with Giemsa (Sigma-Aldrich; Merck
$\mathrm{KGaA}$ ) at room temperature for $20 \mathrm{~min}$. Stained cells were visualized using a light microscope (magnification, x400; Olympus Corporation).

Statistical analysis. Statistical analysis was performed using SPSS 22.0 software (IBM Corp.) and all data are presented as the mean \pm SD of three experimental repeats. Differences between two groups were determined using Student's t-test; differences between multiple groups were determined using one-way ANOVA with Fisher's Least Significant Difference post hoc test. $\mathrm{P}<0.05$ was considered to indicate a statistically signifcant difference.

\section{Results}

SOX2 expression levels are increased in ATC tissue and cell lines. The mRNA expression levels of SOX2 were significantly higher in ATC tissue and cell lines compared with the NC tissue $(\mathrm{P}<0.01$; Fig. $1 \mathrm{~A})$ and Nthy-ori $3-1$ cells $(\mathrm{P}<0.05$; Fig. 1B), respectively. IHC analysis demonstrated that SOX2 protein expression levels were also increased in the ATC tissue compared with the NC tissue (Fig. 1C). Overall, these results suggested that the expression levels of SOX2 may be increased in ATC, indicating that SOX2 may be involved in the development of ATC.

SOX2 promotes ATC cell viability. To determine the function of SOX2 in ATC cell lines, SOX2 was knocked down with siRNA or overexpressed using cDNA constructs in the FRO ATC cell line. Western blotting was used to confirm SOX2 expression levels in FRO cells following the transfections; SOX2 expression levels were significantly decreased in cells transfected with siSOX2 compared with the siNC-transfected cells $(\mathrm{P}<0.01)$, whereas levels were significantly increased in FRO cells transfected with pcDNA3.1-SOX2 compared with pcDNA3.1 ( $\mathrm{P}<0.01$; Fig. 2A). A CCK-8 assay was performed to evaluate the influence of SOX2 on cell viability. In FRO cells transfected with siSOX2, the viability was significantly decreased compared with cells transfected with siNC $(\mathrm{P}<0.01$; Fig. 2B). Conversely, the cell viability was significantly increased in cells transfected with pcDNA3.1-SOX2 compared with pcDNA3.1-transfected cells $(\mathrm{P}<0.05$; Fig. 2B). The effect of SOX 2 on cell proliferation was evaluated using a colony formation assay. Cells transfected with siSOX2 exhibited a significantly lower proliferative ability compared with siNC-transfected cells $(\mathrm{P}<0.05$; Fig. 2C), whereas cells transfected with pcDNA3.1-SOX2 demonstrated significantly enhanced proliferative abilities compared with pcDNA3.1-transfected cells $(\mathrm{P}<0.05$; Fig. 2C). Overall, these results indicated that SOX2 may promote ATC cell proliferation and viability.

SOX2 promotes ATC cell migration and invasion. The effects of SOX 2 on the migratory and invasive abilities of ATC cell lines in vitro were analyzed using wound healing and Matrigel Transwell assays, respectively. siSOX2-transfected cells were observed to have significantly decreased migratory ability compared with siNC-transfected cells, whereas the overexpression of SOX2 with pcDNA3.1-SOX2 significantly enhanced the migratory ability of FRO cells compared with the 

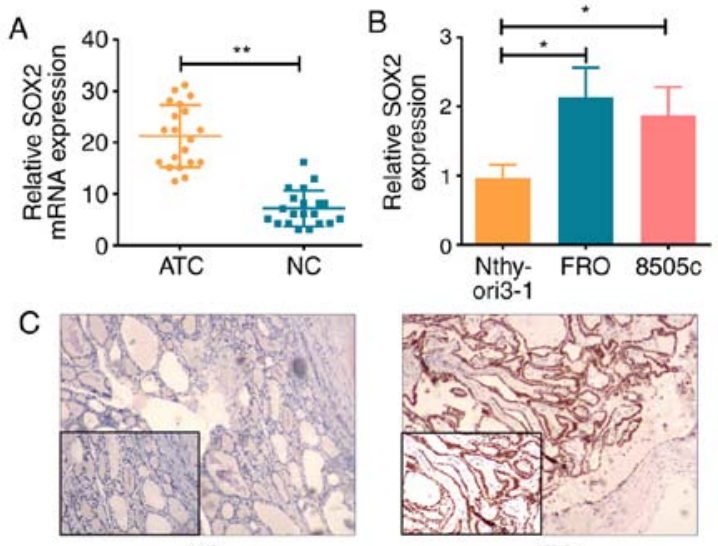

NC

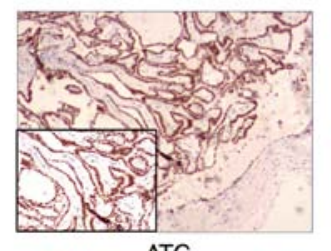

ATC

Figure 1. SOX2 expression levels are increased in ATC tissues and cell lines (A) SOX2 mRNA expression levels in ATC patient tissues and para-carcinoma NC tissues detected by RT-qPCR analysis. (B) SOX2 mRNA expression levels in ATC cell lines (FRO and 8505c) and normal human thyroid follicular epithelial cell line Nthy-ori3-1 detected by RT-qPCR analysis. (C) SOX2 protein expression levels in ATC patient tissues and NC tissues detected by immunohistochemistry (magnification, $\mathrm{x} 100$ ). ${ }^{*} \mathrm{P}<0.05,{ }^{* *} \mathrm{P}<0.01 ; \mathrm{n}=3$ experiments. ATC, anaplastic thyroid cancer; SOX2, SRY-related HMG box-2; $\mathrm{NC}$, negative control; RT-qPCR, reverse transcription-quantitative PCR.

pcDNA3.1 transfected cells (Fig. 3A). In addition, the genetic knockdown of SOX2 with siSOX2 significantly inhibited cell invasion compared with siNC-transfected cells, whereas the overexpression of SOX2 with pcDNA3.1-SOX2 significantly increased the invasive ability of FRO cells compared with pcDNA3.1-transfected cells (Fig. 3B). Overall, these results indicated that SOX2 may promote the migration and invasion of ATC cells.

SOX2 targets FN1 to promote aggressive phenotypes in vitro. To clarify the possible mechanism of the SOX2-mediated increase in the aggressive phenotypes observed in FRO cells, western blotting was used to assess the effect of SOX2 expression on tumor proliferation-related signaling proteins. siSOX2-transfected cells significantly decreased FN1 and p65 expression levels compared with the siNC group, whereas the overexpression of SOX2 in pcDNA3.1-SOX2 cells significantly increased FN1 and p65 expression levels compared with the pcDNA3.1-transfected cells (Fig. 4). Similarly, the genetic knockdown of SOX2 in FRO cells significantly decreased the phosphorylation levels of PI3K and AKT compared with siNC-transfected cells, whereas pcDNA3.1-SOX2-transfected cells demonstrated significantly increased phosphorylation levels of PI3K and AKT compared with pcDNA3.1-transfected cells (Fig. 4). Overall, these results indicated that SOX2 may promote aggressive phenotypes in ATC cell lines by upregulating FN1 expression levels through the activation of the $\mathrm{PI} 3 \mathrm{~K} / \mathrm{AKT}$ signaling pathway.

\section{Discussion}

Previous studies have reported that SOX2 expression levels were increased in numerous types of cancer, including glioblastoma, ovarian cancer, small cell lung cancer and squamous cell carcinoma, which affected cancer cell physiology owing to the involvement of SOX2 in numerous protein-protein interactions and complex cell signaling pathways, such as the Hippo, Hedgehog and Ras Homolog C/Rho-Associated Coiled-Protein Kinase signaling pathways (21-24). Thus, the pathogenic function of SOX2 in multiple types of cancer is of interest. It is reported that FN1 is a direct target of SOX2 in ovarian cancer cells (17), however, the role of SOX2 and its relationship with FN1 have not been investigated in ATC. Given that FN1 expression levels were increased in thyroid cancer (15), it was hypothesized that SOX2 may also regulate the expression of FN1 to mediate the migration and invasion of cells in ATC. Thus, to the best of our knowledge, the present study was the first to focus on the role of SOX2 in ATC cells, including its potential regulatory mechanism. The results demonstrated that SOX2 was overexpressed in ATC tissues and cell lines, which subsequently increased the viability, and proliferative, migratory and invasive ability of ATC cells in vitro. Moreover, SOX2 was found to increase the expression levels of $\mathrm{FN} 1$ and other proteins involved in the PI3K/AKT signaling pathway, including p65, PI3K and AKT. These proteins serve important roles in tumorigenesis and regulate critical cellular functions including survival, proliferation, apoptosis and metabolism (25); for example, the PI3K/AKT signaling pathway has been implicated in modulating a more aggressive phenotype through regulating cholesterol ester formation in prostate cancer cells (26); and PI3K/AKT signaling also been observed to reduce apoptosis, stimulate cell growth and increase proliferation (27). Notably, a previous study also demonstrated that FN1 induced cell proliferation and inhibited apoptosis through the PI3K/AKT signaling pathway (16). Thus, the present study presented a possible model of SOX2-mediated gene regulation in ATC cell lines, in which the overexpression of SOX2 may promote FN1 expression through the PI3K/AKT signaling pathway to induce the aggressive phenotype of ATC.

SOX2 is a pluripotency-associated transcription factor that induces the proliferation, migration, metastasis and invasion of cancer stem cells through various signaling pathways depending on the cancer type (28). Previously, the overexpression of SOX2 has been observed in various types of tumor, but it has not been reported in ATC. In the present study, SOX2 was overexpressed in ATC tissue, FRO and 8505c cell lines, which was in agreement with previous studies into other types of cancer $(17,29)$. To determine the effect of SOX2 on the aggressive cell phenotype, SOX2 expression was overexpressed using cDNA constructs or knocked down using siRNA in FRO cell lines. The results demonstrated that cell activity, migration and invasion were significantly enhanced by SOX2 overexpression and inhibited by SOX 2 knockdown. Therefore, the present study concluded that SOX2 overexpression in ATC may regulate the proliferation, migration and invasion of ATC cells.

FN1 promoted tumor invasion and metastasis through activating specific matrix metalloproteinases (MMPs) in breast cancer (30). SOX2/FN1 signaling pathway is an important pathway mediating the migration and invasion of ovarian cancer cells; SOX2 directly targeted FN1 to induce the expression of MMP-2 and MMP-9 (17). In the present study, FN1 expression levels were increased in SOX2-overexpressed cells, and the protein expression levels of tumor proliferation-related signaling pathways, including p65, p-PI3K/PI3K, $\mathrm{AKT} / \mathrm{p}-\mathrm{AKT}$, were also increased by SOX2. SOX2 induced 

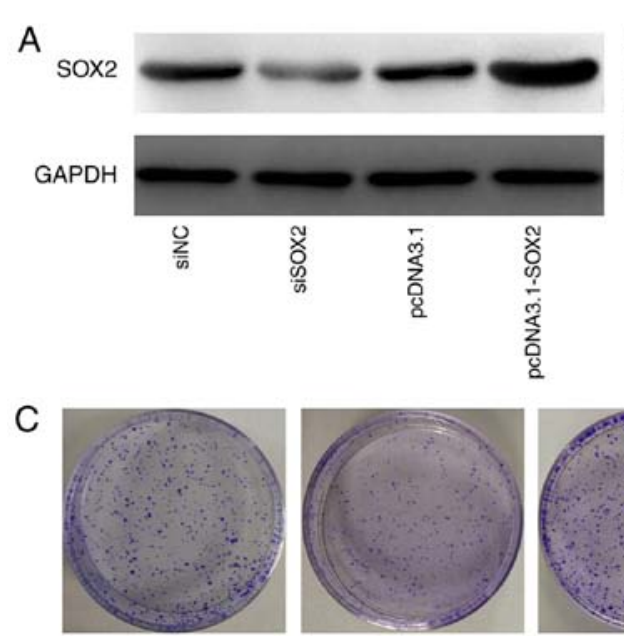

siNC

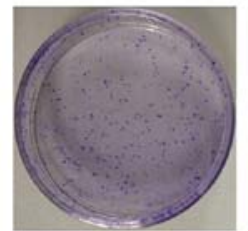

siSOX2

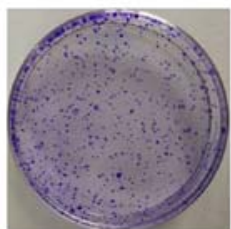

pcDNA3.1
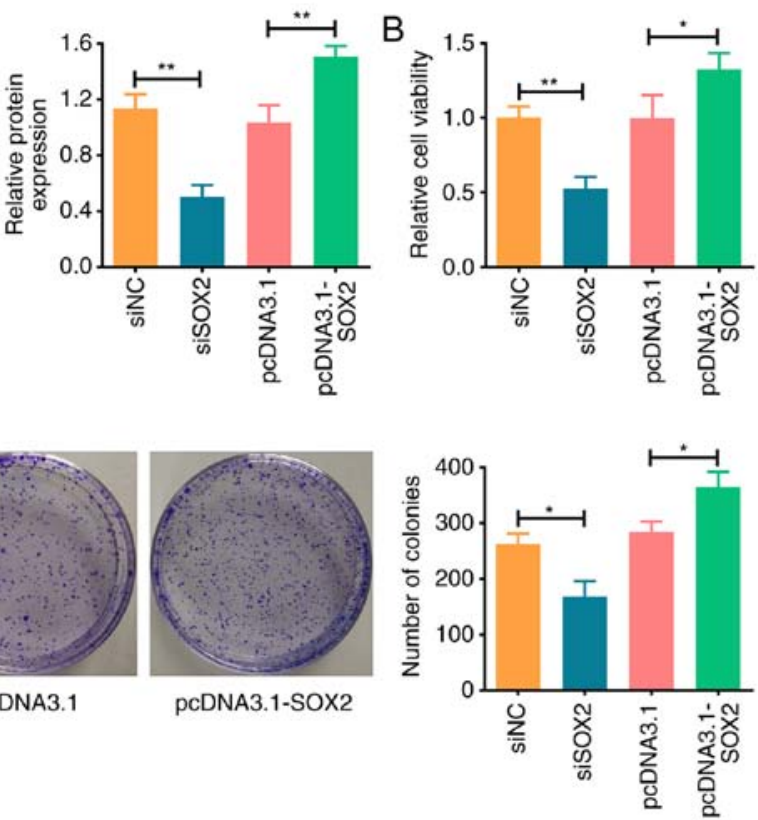

Figure 2. SOX2 promotes the proliferation of anaplastic thyroid cancer cell line FRO. (A) Expression levels of SOX2 in FRO cells transfected with siNC, siSOX2, pcDNA3.1 or pcDNA3.1-SOX2, determined by western blotting. (B) Cell viability was detected using a Cell Counting Kit-8 assay in FRO cells transfected with siSOX2 or pcDNA3.1-SOX2 compared with their respective NCs. (C) Cell proliferation was detected using a colony formation assay in siSOX2- or pcDNA3.1-SOX2-transfected FRO cells compared with their respective NCs. ${ }^{*} \mathrm{P}<0.05,{ }^{* *} \mathrm{P}<0.01 ; \mathrm{n}=3$ experiments. SOX2, SRY-related HMG box-2; NC, negative control; si, small interfering RNA.
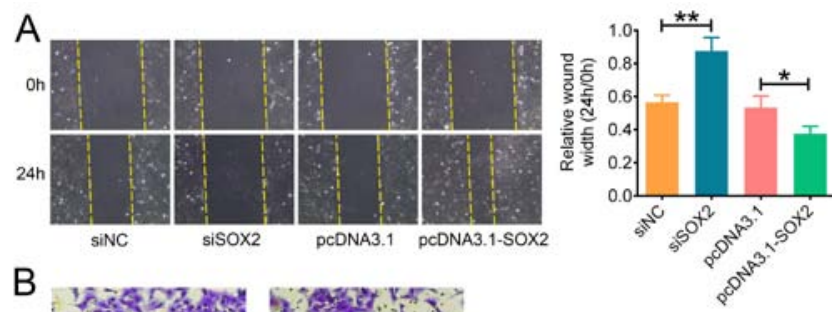

B

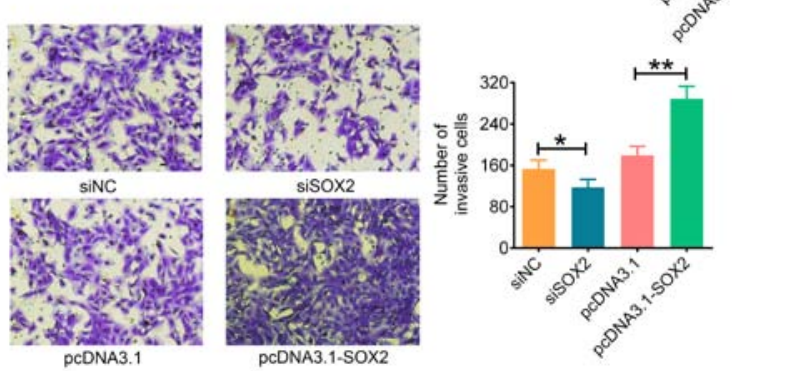

Figure 3. SOX2 promotes the migration and invasion of anaplastic thyroid cancer cell line FRO. (A) Cell migration was detected using a wound healing assay in siSOX2- or pcDNA3.1-SOX2-transfected FRO cells compared with their respective NCs (magnification, x100). (B) Cell invasion was detected using a Matrigel Transwell assay in siSOX2- or pcDNA3.1-SOX2-transfected FRO cells compared with their respective NCs (magnification, $\mathrm{x} 200) .{ }^{*} \mathrm{P}<0.05,{ }^{* * *} \mathrm{P}<0.01 ; \mathrm{n}=3$ experiments. SOX2, SRY-related HMG box-2; $\mathrm{NC}$, negative control; si, small interfering RNA.

the activation of AKT and PI3K by phosphorylation, indicating that SOX2 may mediate FN1 upregulation through the activation of the PI3K/AKT signaling pathway. This is similar to a previous study by Liao et al (31), which reported that FN1 stimulated glioma growth, invasion and survival through the activation of the PI3K/AKT signaling pathway. Besides, p65 as a subunit of NF- $\mathrm{KB}$ was also upregulated by SOX2; it is widely accepted that NF- $\mathrm{KB}$ contributes to the metastasis and invasion of cancer stem cells (32), and it is hypothesized that
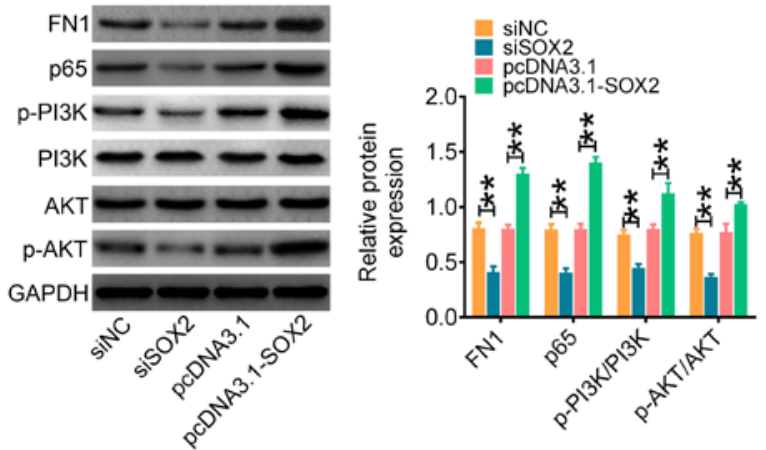

Figure 4. SOX2 expression affects the expression levels of tumor proliferation-related proteins. Protein expression levels of FN1, p65, p-PI3K, PI3K, $\mathrm{AKT}$ and $\mathrm{p}-\mathrm{AKT}$ were assessed using western blotting in siSOX 2 and pcDNA3.1-SOX2 transfected cells. GADPH was used as the loading control. ${ }^{* *} \mathrm{P}<0.01 ; \mathrm{n}=3$ experiments. FN1, fibronectin $1 ; \mathrm{p}$, phosphorylated; si, small interfering RNA; NC, negative control; SOX2, SRY-related HMG box-2.

SOX2 may also mediate FN1 upregulation through activating $\mathrm{NF}-\kappa \mathrm{B}$ signaling. For example, FN1 was observed to induce an aggressive phenotype following the activation of the NF- $\mathrm{KB}$ pathway in nasopharyngeal carcinoma (33). These results illustrated that the overexpression of SOX2 promoted FN1 expression mainly through the PI3K/AKT/NF- $\mathrm{\kappa B}$ signaling pathway, which may induce the aggressive phenotype of ATC.

In conclusion, to the best of our knowledge, this is the first study to report on the function of SOX2 in promoting aggressive phenotypes in ATC and its possible regulatory mechanisms; the present study identified a possible model of SOX2-mediated gene regulation in ATC cell lines. These findings suggested that the observed overexpression of SOX2 in ATC tissues and cell lines may promote the aggressive phenotypes of ATC cells by inducing FN1 expression through 
the PI3K/AKT signaling pathway. These finding provided crucial molecular insights into ATC pathogenesis and indicated that SOX2 may be useful as a therapeutic target for ATC. Investigations on the detailed molecular mechanism of SOX2 in ATC remains to be further researched in the future.

\section{Acknowledgements}

Not applicable.

\section{Funding}

The present study was supported by funding obtained from The Major Science and Technology Projects of Zhejiang Medical and Health Program (grant nos. WKJ-ZJ-1712 and 2015ZDA007) and The General Research Projects of Zhejiang Medical and Health Program (grant nos. 2018KY310, 2017KY029 and 2015KYA036).

\section{Availability of data and materials}

All data generated or analyzed during this study are included in this published article.

\section{Authors' contributions}

PW and JS designed all the experiments and revised the paper. JZ, KW and LG performed the experiments. JG and WW analyzed and interpreted the data. All liabilities related to the content of this article will be borne by the authors.

\section{Ethics approval and consent to participate}

The study was performed in accordance with the Helsinki Declaration and it was approved by The Ethics Committee of Zhejiang Cancer Hospital (Hangzhou, China). Written informed consent was obtained from each participant.

\section{Patient consent for publication}

Not applicable.

\section{Competing interests}

The authors declare that they have no competing interests.

\section{References}

1. Kitahara CM and Sosa JA: The changing incidence of thyroid cancer. Nat Rev Endocrinol 12: 646-653, 2016.

2. Qu Y, Zhuo L, Li N, Hu Y, Chen W, Zhou Y, Wang J, Tao Q, Hu J, Nie $X$ and Zhan S: Prevalence of post-stroke cognitive impairment in china: A community-based, cross-sectional study. PLoS One 10: e0122864, 2015.

3. Smallridge RC, Ain KB, Asa SL, Bible KC, Brierley JD, Burman KD, Kebebew E, Lee NY, Nikiforov YE, Rosenthal MS, et al: American Thyroid Association guidelines for management of patients with anaplastic thyroid cancer. Thyroid 22: 1104-1139, 2012.

4. Giuffrida D and Gharib H: Anaplastic thyroid carcinoma: Current diagnosis and treatment. Ann Oncol 11: 1083-1089, 2000.

5. Rao SN, Zafereo M, Dadu R, Busaidy NL, Hess K, Cote GJ, Williams MD, William WN, Sandulache V, Gross N, et al Patterns of treatment failure in anaplastic thyroid carcinoma. Thyroid 27: 672-681, 2017.
6. Wuebben EL and Rizzino A: The dark side of SOX2: Cancer-a comprehensive overview. Oncotarget 8: 44917-44943, 2017.

7. Sanada Y, Yoshida K, Ohara M, Oeda M, Konishi K and Tsutani Y: Histopathologic evaluation of stepwise progression of pancreatic carcinoma with immunohistochemical analysis of gastric epithelial transcription factor SOX2: Comparison of expression patterns between invasive components and cancerous or nonneoplastic intraductal components. Pancreas 32: 164-170, 2006.

8. Li XL, Eishi Y, Bai YQ, Sakai H, Akiyama Y, Tani M, Takizawa T, Koike M and Yuasa Y: Expression of the SRY-related HMG box protein SOX2 in human gastric carcinoma. Int J Oncol 24: 257-263, 2004.

9. Sattler HP, Lensch R, Rohde V, Zimmer E, Meese E, Bonkhoff H, Retz M, Zwergel T, Bex A, Stoeckle M and Wullich B: Novel amplification unit at chromosome $3 \mathrm{q} 25-\mathrm{q} 27$ in human prostate cancer. Prostate 45: 207-215, 2000.

10. Chen Y, Shi L, Zhang L, Li R, Liang J, Yu W, Sun L, Yang X, Wang Y, Zhang Y and Shang Y: The molecular mechanism governing the oncogenic potential of SOX2 in breast cancer. J Biol Chem 283: 17969-17978, 2008.

11. Alonso MM, Diez-Valle R, Manterola L, Rubio A, Liu D, Cortes-Santiago N, Urquiza L, Jauregi P, Lopez de Munain A, Sampron N, et al: Genetic and epigenetic modifications of Sox2 contribute to the invasive phenotype of malignant gliomas. PLoS One 6: e26740,2011.

12. Carina V, Zito G, Pizzolanti G, Richiusa P, Criscimanna A, Rodolico V, Tomasello L, Pitrone M, Arancio W and Giordano C: Multiple pluripotent stem cell markers in human anaplastic thyroid cancer: The putative upstream role of SOX2. Thyroid 23: 829-837, 2013.

13. Dooley TP, Reddy SP, Wilborn TW and Davis RL: Biomarkers of human cutaneous squamous cell carcinoma from tissues and cell lines identified by DNA microarrays and qRT-PCR. Biochem Biophys Res Commun 306: 1026-1036, 2003.

14. da Silveira Mitteldorf CA, de Sousa-Canavez JM, Leite KR, Massumoto C and Camara-Lopes LH: FN1, GALE, MET, and QPCT overexpression in papillary thyroid carcinoma: Molecular analysis using frozen tissue and routine fine-needle aspiration biopsy samples. Diagn Cytopathol 39: 556-561, 2011.

15. Sponziello M, Rosignolo F, Celano M, Maggisano V, Pecce V, De Rose RF, Lombardo GE, Durante C, Filetti S, Damante $\mathrm{G}$, et al: Fibronectin-1 expression is increased in aggressive thyroid cancer and favors the migration and invasion of cancer cells. Mol Cell Endocrinol 431: 123-132, 2016.

16. Han SW and Roman J: Fibronectin induces cell proliferation and inhibits apoptosis in human bronchial epithelial cells: Pro-oncogenic effects mediated by PI3-kinase and NF-kappaB. Oncogene 25: 4341-4349, 2006.

17. Lou X, Han X, Jin C, Tian W, Yu W, Ding D, Cheng L, Huang B, Jiang $\mathrm{H}$ and Lin B: SOX2 targets fibronectin 1 to promote cell migration and invasion in ovarian cancer: New molecular leads for therapeutic intervention. OMICS 17: 510-518, 2013.

18. Livak JK and Schmittgen TD: Analysis of relative gene expression data using quantitative PCR and the 2(-Delta Delta C(T)) method. Methods 25: 402-408, 2001.

19. He WP, Zhou J, Cai MY, Xiao XS, Liao YJ, Kung HF, Guan XY, Xie D and Yang GF: CHD1L Protein is overexpressed in human ovarian carcinomas and is a novel predictive biomarker for patients survival. BMC Cancer 12: 437, 2012.

20. Repesh LA: A new in vitro assay for quantitating tumor cell invasion. Invasion Metastasis 9: 192-208, 1989.

21. Wen Y, Hou Y, Huang Z, Cai J and Wang Z: SOX2 is required to maintain cancer stem cells in ovarian cancer. Cancer Sci 108: 719-731, 2017.

22. Basu-Roy U,Bayin NS, Rattanakorn K, Han E, Placantonakis DG, Mansukhani A and Basilico C: Sox2 antagonizes the Hippo pathway to maintain stemness in cancer cells. Nat Commun 6: 6411, 2015.

23. Kar S, Sengupta D, Deb M, Pradhan N and Patra SK: SOX2 function and Hedgehog signaling pathway are co-conspirators in promoting androgen independent prostate cancer. Biochim Biophys Acta Mol Basis Dis 1863: 253-265, 2017.

24. Zheng J, Xu L, Pan Y, Yu S, Wang H, Kennedy D and Zhang Y: Sox 2 modulates motility and enhances progression of colorectal cancer via the Rho-ROCK signaling pathway. Oncotarget 8: 98635-98645, 2017. 
25. Yang SX, Polley E and Lipkowitz S: New insights on PI3K/AKT pathway alterations and clinical outcomes in breast cancer. Cancer Treat Rev 45: 87-96, 2016.

26. Yue S,Li J,Lee SY,Lee HJ, Shao T, Song B, Cheng L, Masterson TA, Liu X, Ratliff TL and Cheng JX: Cholesteryl ester accumulation induced by PTEN loss and PI3K/AKT activation underlies human prostate cancer aggressiveness. Cell Metab 19: 393-406, 2014.

27. Chang F, Lee JT, Navolanic PM, Steelman LS, Shelton JG, Blalock WL, Franklin RA and McCubrey JA: Involvement of PI3K/Akt pathway in cell cycle progression, apoptosis, and neoplastic transformation: A target for cancer chemotherapy. Leukemia 17: 590-603, 2003.

28. Weina $\mathrm{K}$ and Utikal J: SOX2 and cancer: Current research and its implications in the clinic. Clin Transl Med 3: 19, 2014.

29. Gut A, Moch $\mathrm{H}$ and Choschzick M: SOX2 gene amplification and overexpression is linked to HPV-positive vulvar carcinomas. Int J Gynecol Pathol 37: 68-73, 2018.

30. Qian P, Zuo Z, Wu Z, Meng X, Li G, Wu Z, Zhang W, Tan S, Pandey V, Yao Y, et al: Pivotal role of reduced let-7g expression in breast cancer invasion and metastasis. Cancer Res 71 6463-6474, 2011.
31. Liao YX, Zhang ZP, Zhao J and Liu JP: Effects of fibronectin 1 on cell proliferation, senescence and apoptosis of human glioma cells through the PI3K/AKT signaling pathway. Cell Physiol Biochem 48: 1382-1396, 2018.

32. Rinkenbaugh AL and Baldwin AS: The NF- $\kappa$ B pathway and cancer stem cells. Cells 5: E16, 2016.

33. Wang J, Deng L, Huang J, Cai R, Zhu X, Liu F, Wang Q, Zhang J and Zheng Y: High expression of Fibronectin 1 suppresses apoptosis through the NF- $\mathrm{BB}$ pathway and is associated with migration in nasopharyngeal carcinoma. Am J Transl Res 9: 4502-4511, 2017.

(i) $\odot$ This work is licensed under a Creative Commons Attribution-NonCommercial-NoDerivatives 4.0 International (CC BY-NC-ND 4.0) License. 